\title{
Prospects for multiwavelength polarization observations of GRB afterglows and the case GRB 030329
}

\author{
S. Klose ${ }^{1}$, E. Palazzi ${ }^{2}$, N. Masetti ${ }^{2}$, B. Stecklum ${ }^{1}$, J. Greiner ${ }^{3}$, D. H. Hartmann ${ }^{4}$, and H. M. Schmid ${ }^{5}$ \\ 1 Thüringer Landessternwarte Tautenburg, 07778 Tautenburg, Germany \\ 2 Istituto di Astrofisica Spaziale e Fisica Cosmica, Sezione di Bologna, CNR, via Gobetti 101, 40129 Bologna, Italy \\ 3 Max-Planck-Institut für extraterrestische Physik, 85741 Garching, Germany \\ ${ }^{4}$ Clemson University, Department of Physics and Astronomy, Clemson, SC 29634, USA \\ 5 Institut für Astronomie, ETH Zürich, 8092 Zürich, Switzerland
}

Received 13 January 2004 / Accepted 21 March 2004

\begin{abstract}
We explore the prospects for simultaneous, broad-band, multiwavelength polarimetric observations of GRB afterglows. We focus on the role of cosmic dust in GRB host galaxies on the observed percentage polarization of afterglows in the optical/near-infrared bands as a function of redshift. Our driving point is the afterglow of GRB 030329, for which we obtained polarimetric data in the $R$ band and $K$ band simultaneously $\sim 1.5$ days after the burst. We argue that polarimetric observations can be very sensitive to dust in a GRB host, because dust can render the polarization of an afterglow wavelength-dependent. We discuss the consequences for the interpretation of observational data and emphasize the important role of very early polarimetric follow-up observations in all bands, when afterglows are still bright, to study the physical properties of dust and magnetic fields in high- $z$ galaxies.
\end{abstract}

Key words. gamma rays: bursts - polarization; dust - extinction

\section{Introduction}

Various independent studies of GRB afterglows have shown that in the optical bands the degree of linear polarization is usually rather low, in the order of 1 to $2 \%$ (for references see, e.g., Björnsson 2003; Covino et al. 2002, 2003; Greiner et al. 2003a; Lazzati et al. 2003; Masetti et al. 2003). If this turns out to be typical of optical afterglows (of long bursts), then the contribution of the dust in GRB host galaxies to the observed polarization properties of the afterglows may not be negligible. While dust in a GRB host galaxy will also manifest itself by changing the spectral energy distribution of the afterglow light away from an original power-law (e.g., Palazzi et al. 1998; Ramaprakash et al. 1998), polarization provides another independent method to constrain the existence of a dust component. If the sources responsible for the long bursts are located in star-forming regions, cosmic dust in the GRB environment will imprint a signal on the polarization properties of the afterglow light in the optical/near-infrared (NIR) bands. One might then imagine a situation in which the polarization by intervening dust (which might be time-independent) can dominate over the intrinsic polarization of the afterglow light. Motivated by our simultaneous optical and NIR polarimetric observations of the afterglow of GRB 030329, we explore here how dust in the GRB hosts may affect the polarimetry of the

Send offprint requests to: $\mathrm{S}$. Klose, e-mail: klose@tls-tautenburg.de afterglow. Thereby, we concentrate on the wavelength dependence of the degree of linear polarization $P$. While the intrinsic polarization of the afterglow light is expected to be due to synchrotron radiation and, thus, wavelength independent, any additional polarization by dust in the GRB host can introduce a wavelength dependence to the measured $P$. Having this in mind, we basically focus on the redshift effect, which modifies this potential wavelength dependence of the degree of polarization (Serkowski 1973; Serkowski et al. 1975).

Recently, Lazzati et al. (2003) have discussed the potential imprint of cosmic dust in GRB hosts on the interpretation of polarization data, with particular emphasis on the afterglow of GRB 021004. Whereas these authors concentrated on the interpretation of the time evolution of the percentage polarization, $P(t)$, here we focus on the wavelength dependence of $P$ at a fixed time. The latter comes into play if dust dominates the polarimetric properties of the afterglow light. While the time evolution of $P$ is basically an indicator for the GRB outflow geometry (Ghisellini \& Lazzati 1999; Sari 1999; Rossi et al. 2004; Lazzati et al. 2004; for the cannonball model see Dado et al. 2004), the wavelength dependence of $P$ is a signature for the presence of dust in the GRB host. Also, whereas a measure of $P(t)$ requires an intense monitoring of the rapidly fading afterglow over many days, the wavelength dependence of $P$ can be investigated at any time, in particular when the afterglow is still in its earliest evolutionary phase, i.e., when the afterglow is brightest. Rapid multiwavelength polarimetric observations of 
GRB afterglows could then allow us to correct for the influence of dust once the time evolution of $P$ is studied.

\section{Theoretical approach}

Our basic assumption is that on average the dust properties in the GRB host galaxy resemble those of the dust in our Galaxy. Though this remains to be proven, currently it is the best approach for such a study. In order to describe the wavelength dependence of the percentage polarization due to dust in the GRB host at a redshift $z$, we adopt the empirical Serkowski law, which for a redshifted host in the observer frame reads

$P(\lambda ; z)=P_{\max } \exp \left(-K \ln ^{2}\left((1+z) \lambda_{\max }^{\text {host }} / \lambda\right)\right)$.

We set $K=(1.86 \pm 0.09) \lambda_{\max }^{\text {host }}(\mu \mathrm{m})-(0.10 \pm 0.05)$ (Wilking et al. 1982). In Eq. (1) $\lambda$ stands for the wavelength of observation and $P_{\max }$ is the maximum percentage polarization at the corresponding wavelength $\lambda_{\max }^{\text {host }}$ in the host. Following Serkowski et al. (1975), we set

$\lambda_{\max }^{\text {host }}(\mu \mathrm{m})=R_{V} / 5.5$,

where $R_{V}$ is the ratio of total-to-selective extinction. In the case of spectropolarimetric observations of a dust-affected afterglow, the observer would simply find a redshifted (and stretched) Serkowski law (Eq. (1)). For broad-band photometric observations in a certain band $X$, however, the percentage polarization as a function of redshift $z$ is given by

$P(X ; z)=\frac{\int_{0}^{\infty} \mathrm{d} \lambda S_{X}(\lambda) F(\lambda) P(\lambda ; z)}{\int_{0}^{\infty} \mathrm{d} \lambda S_{X}(\lambda) F(\lambda)}$.

Here $\lambda=\lambda_{\text {host }}(1+z)$ is the wavelength of observation, $F(\lambda)$ is the observed flux density of the afterglow per unit wavelength, and $S_{X}(\lambda)$ the filter response function. For $F(\lambda)$ we adopt a synchrotron spectrum modified by extinction in the host: $F(\lambda) \propto \lambda_{\text {host }}^{\beta} \exp \left(-\tau\left(\lambda_{\text {host }}\right)\right)$, where $\beta$ is the spectral index, $\tau$ is the wavelength dependent optical depth in the host. When calculating the latter for a given visual extinction $A_{V}$ (host), we assumed the extinction curve of the Milky Way (cf. Reichart 2001). For the filter response functions, $S_{X}(\lambda)$, we used the data provided by ESO for VLT/FORS1 and VLT/ISAAC.

We further assumed that the position angle of linear polarization due to dust, $\theta$, is wavelength independent. This simplification reflects to some degree our incomplete knowledge about the nature of the star-forming regions in which the burster might be placed. As long as the alignment of the scattering grains in the GRB host galaxy is affected by the global magnetic field acting in the host (cf. Covino et al. 1997), we might expect however that those dust grains which are mainly responsible for the NIR polarization are aligned in the same manner as those which are mainly responsible for the polarization at shorter wavelengths. This assumption is supported by observational data from star-forming regions in our galaxy (e.g., McGregor et al. 1994; Whittet et al. 1994). However, if the line of sight crosses dense clumps in interstellar clouds, $\theta$ can become wavelength-dependent (e.g., Messinger et al. 1997).

The synchrotron origin of the afterglow light implies that it is linearly polarized. For our purpose a strict treatment of the
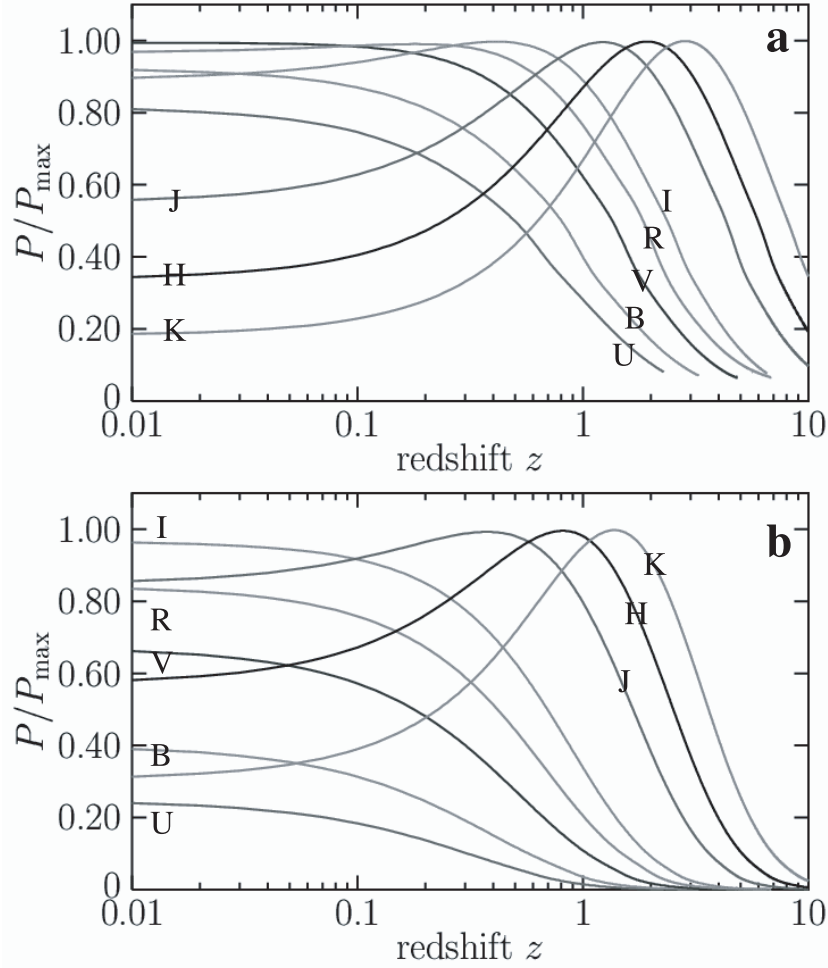

Fig. 1. The theoretically expected dependence of the degree of linear polarization of an intrinsically unpolarized GRB afterglow due to cosmic dust in the GRB host galaxy as a function of redshift for the optical/near-infrared bands (Eq. (3)). Assumed here is a Milky Way extinction law with (a) $R_{V}=3.1$ and (b) $R_{V}=5$ (Eq. (2)). The redshifted $2200 \AA$ feature is only marginally evident in these curves. Nevertheless, we caution that once the redshift shifts the photometric bands into the UV domain the predictions become less secure.

propagation of this radiation is not necessary since its polarization does not depend on wavelength. The subsequent scattering of the light emerging from the afterglow by dust grains will alter its polarization state (leading to an increase or decrease of $P$ depending on both the orientation and shape of the grains) and leave a spectral imprint which is solely due to the dust.

Figure 1 displays the percentage polarization we expect to measure for an intrinsically unpolarized GRB afterglow, if the polarization properties of the afterglow light are caused by dust in the host following a Serkowski law (Eq. (3)), after the correction for the Galactic dust contribution. The degree of linear polarization is plotted as a function of redshift for the optical/NIR bands in units of the maximum polarization $P_{\max }$ measured by the observer at $\lambda_{\max }=(1+z) \lambda_{\max }^{\text {host }}$.

From Fig. 1 several results become apparent. (1) Polarization in the NIR becomes higher than in the optical for redshifts $z \gtrsim 0.5 \ldots 1$. Maximum polarization in the NIR bands occurs at redshifts $z=1$ to 3 . Since most bursts are at redshifts $z \gtrsim 0.5$, NIR polarimetric observations would be more sensitive for dust in the host in basically all cases. (2) Optical versus NIR polarimetry could clearly reveal the presence of cosmic dust in a GRB host galaxy along the line of sight due to the strong differences in the (expected) percentage polarization which cannot be intrinsic to the fireball light. 
(3) If the intrinsic polarization of GRB afterglows is usually rather low at all times, then in some photometric bands the polarization due to dust in the host can dominate (e.g., in $J H K)$, whereas at the same time in other bands the opposite occurs (e.g., $U B V R I)$. In addition, we found that the functional form of $P(\lambda) / P_{\max }$ is not much sensitive on the assumed visual extinction, $A_{V}$ (host) (Eq. (3)), as well as on the spectral index, $\beta$, of the intrinsic afterglow light.

Naturally, these results rely on the assumption of the Serkowski law, including the relation for the parameter $K=$ $K\left(\lambda_{\max }\right)$ and the assumed value for $\lambda_{\max }^{\text {host }}$ (Eq. (2)). In particular, more generalized functions might be used to describe $P(\lambda)$ in the NIR range (e.g., Martin et al. 1999). However, this does not affect the qualitative trends which are evident in Fig. 1.

\section{The case GRB 030329}

Optical polarimetry of the Optical Transient (OT) of GRB 030329 was acquired in the $R$ band with the 2.2-m telescope at Calar Alto (Spain) equipped with the multi-purpose instrument CAFOS. The $2048 \times 2048$ SITe CCD covered a circular field of diameter $16^{\prime}$. A Wollaston prism with rotatable half-wavelength, phase-retarder plate allowed splitting the light from each object in the field into two beams (separated by $\sim 20^{\prime \prime}$ on the CCD frame) which differed for $90^{\circ}$ in their phase angle. By rotating the retarder plate by $45^{\circ}$, one can sample the Stokes' polarization parameters $U$ and $Q$ by using two consecutive images. The quasi-perfect transmission of the Wollaston prism makes two orientations sufficient for linear polarization measurements (Lamy \& Hutsemékers 1999). Using this setup, six pairs of images, with exposure time of $300 \mathrm{~s}$ for each frame, were secured between 22:26 UT of 30 March 2003 and 02:14 UT of 31 March 2003, when the brightness of the afterglow was $R \approx 16.2$. The frames were bias-subtracted and flat-fielded with the standard cleaning procedure, having care of using flat fields collected using the same rotation angle in the retarder plate as of the corresponding GRB field image. PSF fitting as implemented in DAOPHOT II (Stetson 1987) within MIDAS was used for the data analysis. The pairs of images were first analyzed separately, to look for variations in the $U$ and $Q$ values of OT and field stars during the observations. As none were found, we coadded together the images with the same retarder plate angle and considered the average images in order to increase the signal-to-noise ratio of the polarization measurement. In order to correct for the instrumental plus Galactic interstellar medium (ISM) contribution to the total polarization we used a number of bright field stars around the OT and as close as possible to it on the image. To evaluate the Stokes' $U$ and $Q$ parameters of the optical transient emission, and its percentage polarization $P$ and position angle $\theta$, we applied the method described by di Serego Alighieri (1997). The values (not corrected for the ISM contribution) of these parameters for the OT and their average values for the field stars are reported in Fig. 2. The corrected measurement of $U$ and $Q$ from our data gives a polarization percentage $P=1.1 \pm 0.4 \%$ and a polarization angle $\theta=70^{\circ} \pm 11^{\circ}$. This value of $P$ is also corrected for the polarization bias (Wardle \& Kronberg 1974).

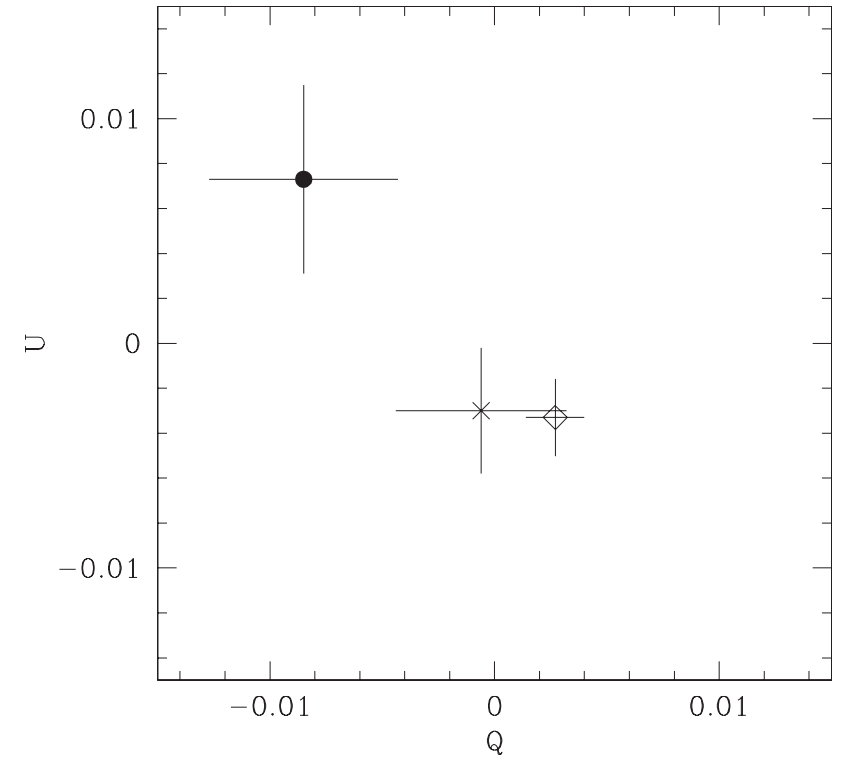

Fig. 2. Plot showing the values of the Stokes' parameters $U$ and $Q$ including their $1 \sigma$ error bars, not corrected for ISM polarization, of the OT of GRB 030329 (marked with a filled dot) and of the average of field stars (cross) in the averaged $R$-band CAFOS data. The latter agrees well with the mean value for $U$ and $Q$ of field stars from the VLT+FORS1 $R$-band campaign on this OT (Greiner et al. 2003a), which is reported as an open diamond. The OT is separated from the region occupied by the field stars: this suggests that it indeed has net intrinsic polarization, albeit at low level.

$K$-band polarimetry of the afterglow of GRB 030329 was performed with the NIR camera Omega Cass mounted at the Calar Alto $3.5 \mathrm{~m}$ telescope on 30.3.2003, UT 22:18-23:18, when the afterglow had a magnitude of $K \approx 14$. Omega Cass is equipped with a Rockwell $1024 \times 1024$ pixel HAWAII array. The camera was used in the wide-field mode $\left(0.3^{\prime \prime} / \mathrm{pixel}\right)$, giving a field of view of $5^{\prime} \times 5^{\prime}$. Again, a Wollaston prism was used. Four polarization sequences were performed, yielding images at values of $0 / 90^{\circ}$ and $45 / 135^{\circ}$ of the position angle, respectively. There was only one comparison star in the field, which was assumed to be intrinsically unpolarized. Thus its net polarization includes polarization due to both the ISM and the instrument, and defines the zero point for the calculation of the afterglow polarization. From Fig. 3 it is obvious that the error of the comparison star which is the brightest star in the field of view of Omega Cass dominates the error budget since it was about $0.3 \mathrm{mag}$ fainter than the afterglow. The final result is $P=2.1 \pm 1.2 \%, \theta=54.1^{\circ} \pm 10.4^{\circ}$ (Fig. 3), after correction for the polarization bias. Even though our measurement error allows for a $P=0$ (within $2 \sigma$ ), we note that the polarization angle we deduce for the $K$ band is basically identical to those found for the $R$ band. This makes us confident that our measurement relies on a real detection and is not an upper limit.

In principle, the amount of the percentage polarization measured in $R$ and $K$ could be used to constrain the extinction in the host along the line of sight using the empirical relation $P_{\max } \lesssim 9 E(B-V)$ found for Galactic dust (Schmidt-Kaler 1958; Serkowski et al. 1975). However, the less known ratio of total-to-selective extinction of the dust in the host, the scatter of 


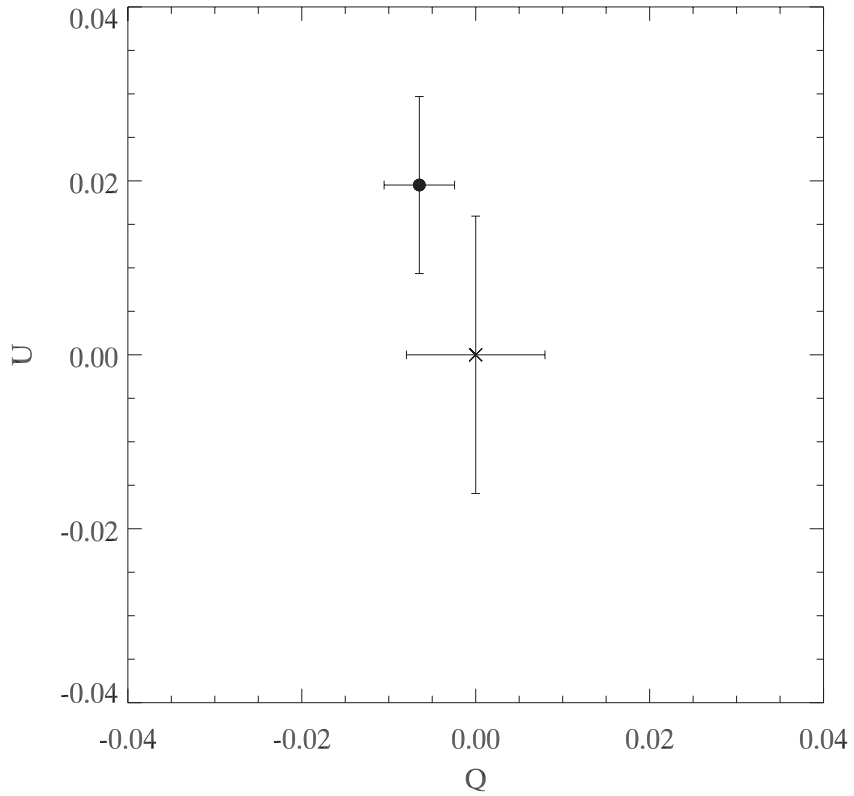

Fig. 3. The deduced Stokes' parameters $U$ and $Q$ of the afterglow light in the $K$ band based on the Omega Cass data. The cross marks the polarization of the field star while the filled dot represents the afterglow. At the time of the observation the flux from the underlying host galaxy and from the supernova component (SN 2003dh) can be fully neglected.

the $P_{\max }$ vs. $E(B-V)$ relation for small $E(B-V)$ (cf. Serkowski et al. 1975), as well as the observed spread in the empirical parameter $K=K\left(\lambda_{\max }\right)$ (Eq. (1); cf. Wilking et al. 1982; Whittet et al. 1992) naturally limits the validity of such an approach.

A qualitative constraint on the impact of dust in the GRB host galaxy on the fireball light can be set by comparing the predicted ratios of the percentage polarization in the different photometric bands (Fig. 1) with the observed ones. For $R_{V}=3.1\left(R_{V}=5\right)$ at the redshift of GRB $030329(z=0.1685$; Greiner et al. 2003b), the predicted ratio of the percentage polarization between the $R$ - and the $K$ band is approximately 4.0 (1.6) if dust dominates, and 1.0 if there is no (aligned) dust. Since the former is not evident in our data (we measure $P_{R} / P_{K}=0.5 \pm 0.3$ ), we conclude that at the time of our observations, $\sim 1.5$ days after the burst, the intrinsic polarization of the afterglow dominates over any polarization possibly introduced by dust in the GRB host galaxy. This conclusion is strengthened by the results of the VLT polarimetry of the afterglow of GRB 030329 (Greiner et al. 2003a). Spectropolarimetric VLT data obtained 1.63 days after the burst, thus nearly simultaneously with the Calar Alto data, do not show evidence for a smooth wavelength dependence of $P$ in the region between 400 and $800 \mathrm{~nm}$ (Fig. 4). The same holds for the spectropolarimetric VLT data taken 0.64, 2.56, and 2.64 days after the burst (Greiner et al., in preparation).

\section{Concluding remarks}

Encouraged by our successful simultaneous optical and NIR polarimetric observations of the afterglow of GRB 030329, we have explored here in which manner

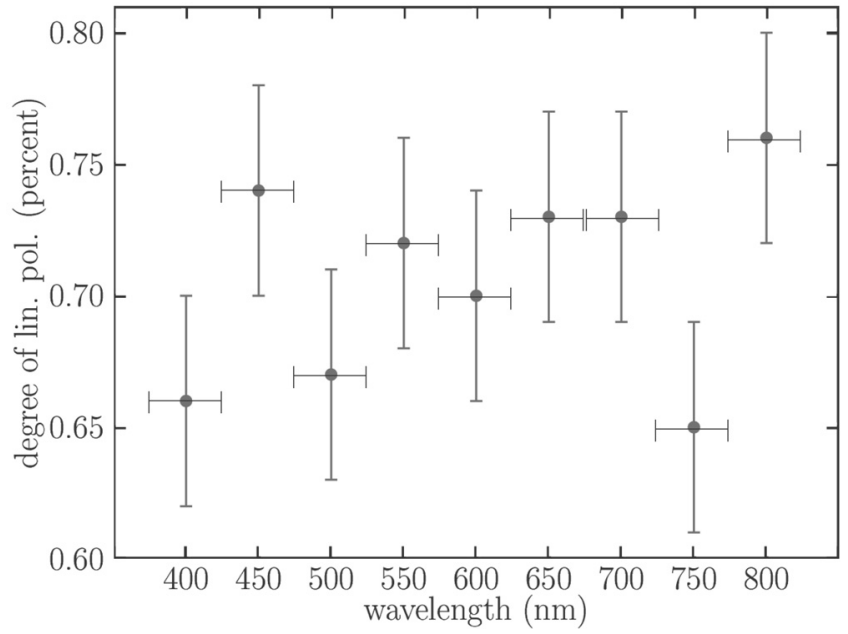

Fig. 4. Result of the spectropolarimetry of the afterglow of GRB 030329 based on data taken with VLT/FORS1 1.63 days after the burst, nearly simultaneously with the Calar Alto data. There is no obvious evidence for a wavelength dependence of $P$.

simultaneous multicolor polarimetric observations can reveal an imprint from cosmic dust in GRB host galaxies on the afterglow light. We have argued that dust in GRB hosts can strongly affect the polarization properties of an afterglow, depending on its redshift and on the photometric band, in which the observations are performed. In particular, the wavelength dependence of polarization due to dust, which is in strong contrast to the intrinsic wavelength independence of the fireball light, could be used as an indicator for the presence of dust.

With the upcoming $S$ wift satellite mission GRB afterglows are expected to be localized within seconds after burst trigger, in principle allowing for first polarimetric observations within the first hour after a burst. Since the amount of maximum polarization, $P_{\max }$, can rapidly increase with increasing visual extinction within the host galaxy (Schmidt-Kaler 1958; Serkowski et al. 1975), in principle large degrees of linear polarization could be detectable for the most intrinsically extinguished afterglows. Conversely, if a wavelength dependent polarization is never found in an afterglow then either the host dust properties must be substantially different from those of the Galactic ISM, or there is no dust at all, or the magnetic field in the host galaxy is weak and/or not well ordered.

The position angle of the polarization defines the orientation of the predominant magnetic field in the host galaxy along the line of sight. This predominant field can either be the large scale magnetic field acting in the GRB host (in particular, if the host is seen edge-on), or the field characterizing the starforming region in which the burster is placed. The detection of interstellar dust extinction in the host without a substantial polarization signal could hint to a strongly tangled magnetic field structure in the host galaxy, or even indicate the absence of a magnetic field large enough $\left(\sim 10^{-8} \mathrm{G}\right)$ to align the dust grains in the host galaxy. Both effects would indicate very strong evolutionary effects in the galactic magnetic field structure as practically all well studied nearby galaxies are known to have 
ordered magnetic field on large ( $\sim \mathrm{kpc}$ ) scales (Kronberg 1994; Scarrott 1996; Wielebinski \& Krause 1993). In the Milky Way the dust grain alignment along the arbitrary line of sights due to the Galactic magnetic field produces a polarization per magnitude of visual extinction in the range $P / A_{\mathrm{V}} \approx 0.6-3 \% \mathrm{mag}^{-1}$ (Serkowski et al. 1995). Thus a polarization below this value might indicate special conditions in a GRB host galaxy, including the star-forming region in which the burster is placed.

Acknowledgements. S.K. acknowledges support from the Deutsche Akademische Austauschdienst (DAAD) under grants No. D/0237747 and D/0103745; N.M. and E.P. acknowledge support under CRUI Vigoni program 31-2002. D.H.H. acknowledges support under NSF grant INT-0128882. This work is partly based on observations at the German-Spanish Astronomical Centre, Calar Alto, operated by the Max-Planck-Institute for Astronomy, Heidelberg, jointly with the Spanish National Commission for Astronomy. We thank the staff at Calar Alto for their assistence, in particular A. Aguirre, M. Alises, S. Pedraz, and U. Thiele. We thank Elena Pian for a careful reading of the manuscript. We thank the referee for a rapid response and helpful remarks.

\section{References}

Björnsson, G. 2003, preprint [arXiv: astro-ph/0302177]

Covino, E., Palazzi, E., Penprase, B. E., Schwarz, H. E., \& Terranegra, L. 1997, A\&AS, 122, 95

Covino, S., Lazzati, D., Malesani, D., et al. 2002, A\&A, 392, 865

Covino, S., Ghisellini, G., Lazzati, D., \& Malesani, D. 2003, preprint [arXiv:astro-ph/0301608]

Dado, S., Dar, A., \& de Rújula, A. 2004, preprint [arXiv: astro-ph/0403015]

di Serego Alighieri, S. 1997, in Instrumentation for Large Telescopes - VII Canary Islands Winter School of Astrophysics, ed. J. M. Rodriguez de Espinosa, A. Herrero, \& F. Sánchez (Cambridge: Cambridge University Press), 287
Ghisellini, G., \& Lazzati, D. 1999, MNRAS, 309, L7

Greiner, J., Klose, S., Reinsch, K., et al. 2003a, Nature, 427, 157

Greiner, J., Peimbert, M., Estabian, C., et al. 2003b, GCN, 2020

Kronberg, P. P. 1994, Rep. Progr. Phys., 57, 325

Lamy, H., \& Hutsemékers, D. 1999, ESO Messenger, 96, 25

Lazzati, D., Covino, S., di Serego Alighieri, S., et al. 2003, A\&A, 410, 823

Lazzati, D., Covino, S., Gorosabel, J., et al. 2004, A\&A, submitted, preprint [arXiv:astro-ph/0401315]

Martin, P. G., Clayton, G. C., \& Wolff, M. J. 1999, ApJ, 510, 905

Masetti, N., Palazzi, E., Pian, E., et al. 2003, A\&A, 404, 465

McGregor, P. J., Harrison, T. E., Hough, J. H., \& Bailey, J. A. 1994, MNRAS, 267, 755

Messinger, D. W., Whittet, D. C. B., \& Roberge, W. G. 1997, ApJ, 487, 314

Palazzi, E., Pian, E., Masetti, N., et al. 1998, A\&A, 336, L95

Ramaprakash, A. N., Kulkarni, S. R., Frail, D. A., et al. 1998, Nature, 393,43

Reichart, D. E. 2001, ApJ, 553, 235

Rossi, E., Lazzati, D., Salmonson, J. D., \& Ghisellini, G. 2004, MNRAS, submitted, preprint [arXiv:astro-ph/0401124]

Sari, R. 1999, ApJ, 524, L43

Scarrott, S. M. 1996, QJRAS, 37, 297

Schmidt-Kaler, Th. 1958, Z. Astrophys., 46, 145

Serkowski, K. 1973, in Interstellar Dust and Related Topics, ed. J. M. Greenberg, \& H. C. van de Hulst (Dordrecht: Reidel), IAU Symp., 52,145

Serkowski, K., Mathewson, D. S., \& Ford, V. L. 1975, ApJ, 196, 261

Stetson, P. B. 1987, PASP, 99, 191

Wardle, J. F. C., \& Kronberg, P. P. 1974, ApJ, 194, 249

Whittet, D. C. B., Martin, P. G., Hough, J. H., et al. 1992, ApJ, 386, 562

Whittet, D. C. B., Gerakines, P. A., Carkner, A. L., et al. 1994, MNRAS, 268, 1

Wielebinski, R., \& Krause, F. 1993, A\&ARv, 4, 449

Wilking, B. A., Lebofsky, M. J., \& Rieke, G. H. 1982, AJ, 87, 695 